\title{
Recent advances in the epidemiology and diagnosis of some common vestibular disorders
}

\author{
Mario Milkov, MD, PhD \\ Department of Dental Material Sciences and Propedeutics of Prosthetic Dental Medicine, \\ Faculty of Dental Medicine, Medical University of Varna
}

\begin{abstract}
There is a rising interest in the diagnosis of the common vestibular disorders in childhood and adulthood. The results from numerous epidemiological investigations prove the socio-medical importance of this pathology. In the present survey, the applications of the main diagnostic methods in this interdisciplinary field are discussed. Here belong the caloric test, the video head impulse test, the cervical and ocular vestibular evoked myogenic potentials, the videonistagmography, the electrococheography, the magnetic resonance imaging, the computed tomography, etc. A special attention is paid to the comparative diagnosis of some socially significant common vestibular disorders.

Key words: vestibular disorders, diagnostic methods, social epidemiology
\end{abstract}

In the last few years, significant advances in the diagnostic capacities for the socially significant vestibular disorders in adults and children have undountedly been achieved.

\section{Epidemiology of common vestibular disorders}

There are numerous epidemiological investigations devoted to the common vestibular disorders worldwide.

Vestibular migraine is one of the most common presenting complaints to physicians in primary care, otolaryngology and neurology (2). Epidemiologic data suggest that it may affect up to $1 \%$ (32) and between $1 \%$ and $3 \%$ of the general population $(2,20,23), 7 \%$ of the patients in dizziness clinics (32) and 10 to $30 \%$ of the patients seeking treatment for dizziness (2).

The prevalence of vestibular disorders in childhood ranges from $0,4 \%$ to $15 \%(8)$. The results from a cross-sectional study of 117 patients at a mean age of 10 years demonstrate that benign paroxysmal vertigo of childhood is the most prevalent diagnosis $(41,9 \%)$ followed by vestibular migraine $(16,2 \%)$.

According to A. Zwergal \& M. Dieterich (40), the eight most frequent vestibular disorders account for more than $70 \%$ of all presentations of vertigo, dizziness, and imbalance. Acute unilateral vestibulopathy and vestibular stroke are most important in acute (and mostly non-repetitive) vestibular disorders; benign paroxysmal positional 
vertigo, Menière's disease and vestibular migraine are the most common episodic vestibulopathies while bilateral vestibulopathy/presbyvestibulopathy, functional dizziness and cerebellar dizziness belong to chronic vestibular disorders. Videooculography and audiometry are used for securing the diagnosis.

Vestibular disorders are diagnosed in 159 out of a total of 203 children at a mean age of $11,16 \pm 3,87$ years (range, $1-17$ years) (in 78,33\% of the cases) among 3400 patients undergoing vestibular assessment at a vertigo center in a tertiary hospital during a three-year period (11). Benign paroxysmal positional vertigo is the most common diagnosis (in 100 children or in $49,26 \%$ ) followed by vestibular migraine (in 83 children or in 40,89\%), benign paroxysmal vertigo of childhood, vestibular neuritis and psychogenic vertigo (in nine children or in $4,43 \%$ of the cases each).

Between September 2010 and September 2018 in an observational, cross-sectional and retrospective study of 212 children at a mean age of 14,5 $\pm 3,9$ years, benign paroxysmal vertigo of childhood, vestibular dysfunction and vestibular migraine account for more than $65 \%$ of the cases (15). In a total of 206 patients at a mean of 8 years 11 months, the most common causes of acute nystagmus are migraine (in 25,7\%) and vestibular disorders (in $14,1 \%$ of the cases) (10).

The prevalence of peripheral vestibular disorders among children aged between 0 and 15 years in Germany is 15,16 per 100000 individuals (14). The prevalence of benign paroxysmal positional vertigo is 10,21 per 100000 individuals, that of vestibular neuritis is 3,5 per 100000 individuals and that of Menière's disease is 1,54 per 100000 individuals. Among 1021 patients at a mean age of $12,5 \pm 4,9$ years (range: 9 months -21 years) examined within a pediatric vestibular program between January 2012 and March 2019, the most common diagnoses include vestibular migraine (in 35,0\%), benign paroxysmal positional vertigo (in $21,6 \%$ ) and persistent postural perceptual dizziness (in $11,2 \%$ of the cases) (36).

In 2016, 59986 patients have been diagnosed with peripheral vestibular disorders with prevalence rate of 2833,4 per 100000 population in Taiwan (38). Prevalence of benign paroxysmal positional vertigo is 446,4 per 100000 population, of vestibular neuritis is 307,2 per 100000 population and of Meniere's disease is 70,4 per 100000 population.
The cross-sectional analysis of the responses from the 2016 National Health Interview Survey in the United States for children aged 3 to 17 years demonstrates dizziness or imbalance in 3,5 million patients at a mean age of 11,5 years; dizziness alone in 1,2 million patients at a mean age of 12,7 years and balance impairment alone in 2.3 million patients at a mean age of 10,6 years (3).

In a total of 2596 visits by 2464 patients to a large interdisciplinary tertiary emergency department, at least one vestibular symptom is reported (12). Vestibular symptoms are the main reason for the consultation in 1677 visits (in $64,60 \%$ of the cases). They are classified as dizziness (in 43,8\%), vertigo (in 33,9\%), postural symptoms (in 6,5\%), or more than one symptom (in 15,8\% of the cases).

The analysis of the cases diagnosed with vestibular disorders in primary care and in a neurotology unit between October 15, 2017 and October 14, 2018 identifies that benign paroxysmal positional vertigo is the most common cause of vestibular symptoms (5). Vestibular migraine and ischemic vestibular symptoms are common in primary care whereas Ménière's disease and undifferentiated episodic vestibular syndrome are common in a specialized centre.

Among a total of 1066 patients at a mean age of $51,1 \pm 15,3$ years at the German Center for Vertigo and Balance Disorders, the following seven most common vestibular disorders are registered: functional dizziness (in 346 patients or in $32,46 \%$ ), vestibular migraine (in 215 patients or in 20,17\%), Menière's disease (in 142 patients or in 13,32\%), benign paroxysmal positional vertigo (in 134 patients or in $12,57 \%$ ), unilateral vestibulopathy (in 114 patients or in $10,69 \%$ ), bilateral vestibulopathy (in 66 patients or in 6,19\%) and vestibular paroxysmia (in 49 patients or in $4,60 \%$ of the cases) (30). The results from a population-based epidemiological survey of a total of 70315919 patients in Germany demonstrate a prevalence rate across all age groups of unspecific vertigo, Menière's disease, benign paroxysmal positional vertigo, vestibular neuritis and other peripheral vestibular disorders of $65 \%$ (6461 cases per 100000 individuals) (13). The prevalence of vertigo is statistically significantly higher in women $(65,4 \%)$ than in men $(34,6 \%)$ $(\mathrm{p}<0,001)$.

In a total of 392 patients at a mean age of $52,39 \pm 13,87$ years (range 11-90 years) visiting the neurological outpatient clinic of the First Affiliated 
Hospital of Chongqing Medical University from January 2016 to June 2017, peripheral vestibular disorders account for $54,6 \%$ of the patients, central vestibular disorders including vestibular migraine account for $22,4 \%$ of the patients and psychogenic vertigo accounts for $16,3 \%$ of the patients (22). Benign paroxysmal positional vertigo is most common in patients aged under 60 years (in 30,8\%) and over 60 years (in 27,9\% of the cases). Vestibular migraine is diagnosed in $14,4 \%$ of younger patients and Menière's disease is detected in $11,7 \%$ of older patients.

\section{Comparative diagnosis of common vestibular disorders}

The abnormalities of the cervical and ocular vestibular evoked myogenic potentials in common disorders of the vestibular system and its pathways are reviewed (33). According to M. Strupp et al. (31), there are already internationally accepted diagnostic criteria for benign positional paroxysmal vertigo, Menière's disease, bilateral vestibulopathy, vestibular paroxysmia, and functional dizziness. The acute and peripheral vestibular syndromes can usually be differentiated based on the history and the clinical examination. 'Cerebellar vertigo' is a clinically important entity.

Taking into consideration the common overlap of vertigo and migraine across vestibular diseases and the fact that vestibular migraine often imitates various vestibular disorders, there is a need for clinical trials focusing on specific laboratory biomarkers including serological, radiological and electrophysiological examinations as well as for development of more detailed and complete diagnostic criteria (16).

The analysis of a total of 610 patients at a mean age of $58,1 \pm 16,3$ years from the outpatient clinic of the German Center of Vertigo and Balance Disorders demonstrates that iPad-based medical device (medx) designed for the diagnosis of vertigo and dizziness has an accuracy for the most common diagnoses between $82,1 \%$ and $96,6 \%$, a sensitivity of $40 \%$ to $80,5 \%$ and a specificity of more than $80 \%$ (9). The sensitivity, specificity, positive and negative predictive values of six most common clinical diagnoses are as follows: bilateral vestibulopathy $(81,6 \% ; 97,1 \% ; 71,1 \%$ and $97,5 \%)$, Menière's disease $(77,8 \% ; 97,6 \% ; 87,0 \%$ and $95,3 \%$ ), benign paroxysmal positional vertigo $(61,7 \% ; 98,3 \% ; 86,6 \%$ and $93,4 \%)$, downbeat nystagmus syndrome $(69,6 \% ; 97,7 \% ; 71,1 \%$ and $97,5 \%)$, vestibular migraine $(34,7 \% ; 97,8 \% ; 76,1 \%$ and $88,3 \%)$ and phobic postural vertigo $(80,5 \%$; $82,5 \% ; 52,5 \%$ and $94,6 \%$ ).

Between January 2010 and March 2017, platform posturography is performed in 202 patients with Ménière's disease, 154 patients with benign paroxysmal positional vertigo and 31 patients with vestibular neuritis during the non-acute phase of vertigo (28). The velocities of body oscillation of benign paroxysmal positional vertigo patients with eyes open and closed are statistically significantly faster than those of Ménière's disease patients $(p<0,001$ and $p=0,033$, respectively) and the velocity of body oscillation of vestibular neuritis patients with eyes open is statistically significantly faster than that of Ménière's disease patients with eyes closed $(p=0,0083)$. There are significant correlations between age and the velocity of body oscillation with both eyes open and closed $(p<0,001)$ as well as between Ménière's disease or vestibular neuritis and the velocity of body oscillation with eyes open $(\mathrm{p}=0,0194)$.

The results of video head impulse test and caloric test demonstrate abnormal caloric tests in 39 patients with Ménière's disease (in 66,1\%) and in 17 patients with vestibular migraine (in $34 \%$ of the cases) (39). There is abnormal video head impulse test gain of the lateral canal in 23 Ménière's disease patients (in 39\%) and in nine vestibular migraine patients (in $18 \%$ of the cases). There is a normal caloric test in $11,9 \%$ of the patients with an abnormal video head impulse test and an abnormal caloric test in $33,9 \%$ of the patients with a normal video head impulse test.

The degree of asymmetry for ocular vestibular evoked myogenic potentials to bone-conducted vibration and cervical vestibular evoked myogenic potentials to air-conducted sound are compared between 100 vestibular migraine and unilateral Menière's disease patients within a retrospective study in an interdisciplinary tertiary center for vertigo and balance disorders (4). The asymmetry ratio of cervical vestibular evoked myogenic potential p13n23 amplitudes is statistically significantly higher for Menière's disease $(0,43 \pm 0,34)$ than for vestibular migraine $(0,26 \pm 0,24$; adjusted $\mathrm{p}=0,0002$ ). Menière's disease patients display a statistically significantly higher asymmetry ratio for cervical vestibular evoked myogenic potential than for ocular vestibular evoked myogenic 
potential amplitudes $(0,43 \pm 0,34$ versus $0,23 \pm 0,22$; $\mathrm{p}<0,0001$ ).

The results from a retrospective cohort of 25 definite Menière's disease patients, 34 definite vestibular migraine patients and 13 control subjects in a multidisciplinary neurootology clinic between January, 2015 and May, 2017 demonstrate statistically significantly lower amplitudes of cervical $(p=0,007)$ and ocular vestibular evoked myogenic potentials $(p<0,001)$ in Menière's disease affected ears than in control ears (25). Vestibular migraine ears have statistically significantly lower ocular vestibular evoked myogenic potential amplitudes $(p=0,001)$ than control ears. Menière's disease affected ears have statistically significantly more absent cervical $(25,9 \%$ versus $0 \% ; \mathrm{p}=0,005)$ and ocular vestibular evoked myogenic potential responses $(40,7 \%$ versus $0 \% ; p<0,001)$ than control ears. The ocular vestibular evoked myogenic potential latency is statistically significantly shorter for both Menière's disease $\mathrm{p}<0,001)$ and vestibular migraine affected ears $p<0,001)$ than for control ears $(25)$.

The results from a prospective study of 32 migraine patients and 31 vestibular migraine ones reveal a numerically higher incidence rate of ears with absent cervical vestibular evoked myogenic potentials in the first group $(\mathrm{p}=0,106)(21)$. There are no statistically significant differences between these groups in terms of the p13 or $n 23$ latency, interpeak amplitude, and amplitude asymmetry ratio in right and left ears $(\mathrm{p}>0,05)$.

A systematic review of 26 articles retrieved from PubMed and comprising a total of 1181 patients among whom there are 296 vestibular neuritis patients, 378 Ménière's disease patients and 507 benign paroxysmal positional vertigo patients proves that cervical and ocular vestibular evoked myogenic potentials are particularly useful in improving the topographic diagnosis of vestibular neuritis (26).

In combination with videonystagmography and video head impulse test, vestibular evoked myogenic potentials characterize vertigo and provide support for the diagnosis of superior semicircular canal dehiscence syndrome, Menière's disease, vestibular neuritis or idiopathic bilateral vestibulopathy (6).

Contemporary diagnosis of vertigo, dizziness and equilibrium disorders is based on a series of methods such as head impulse, nystagmus and test of skew-test, caloric tests, three-dimensional video head impulse test, cervical and ocular vestibular evoked myogenic potentials (27).

Using the caloric test, video head impulse test for all six semicircular canals and vestibular evoked myogenic potentials, persistent postural-perceptual dizziness is diagnosed in 28 out of a total of 147 consecutive patients with dizziness at a mean age of $59,5 \pm 15$ years (in $19,05 \%$ of the cases) (1). The most common initial events are vestibular neuritis(in 39,3\%) and benign paroxysmal positional vertigo (in $10,7 \%$ of the cases). The utility of complete neurophysiological assessment of vestibular function is outlined.

A close presentation dilemma of similarities between benign paroxysmal positional vertigo and vestibular migraine presented by very similar positional nystagmus is examined in 12 relatively uncommon patients comprehensively examined with videonystagmography and brain magnetic resonance imaging with contrast matter (18).

The current status of the most frequently used vestibular tests for vestibular hypofunction as well as for canal and otolith function is comprehensively reviewed (29). Here belong the video head impulse test, the functional head impulse test, the torsion swing test divided into the sinusoidal harmonic acceleration test and the pseudo-random rotation test, the velocity step test as well as the cervical and ocular vestibular evoked myogenic potentials recorded using electromyography.

The symptoms of peripheral and central vestibular dysfunction such as vertigo, nausea and vomiting, intolerance to head motion, spontaneous nystagmus, unsteady gait and postural instability caused by injury to peripheral or central vestibular structures are assessed (7). Vestibular neuronitis is the most common form of acute peripheral vestibular dysfunction.

Within a cross-sectional clinical study of 310 patients and 53 healthy controls, there is a statistically significantly higher prevalence of enhanced vestibulo-ocular reflex responses of video head impulse test in Menière's disease, central origin vertigo and vestibular migraine than other neurootologic diseases and controls (35). These responses as a diagnostic sign of Menière's disease have a sensitivity of $42,59 \%$ and a specificity of $86,32 \%$ with an odds ratio of $4,68(p<0,001)$.

An ambulatory medical device that allows vestibular telemetry to record eye movements over a 30-day period is applied in 16 participants (24). 
The vestibular telemetry reveals distinct differences between the nystagmus produced during an acute Ménière's attack and attacks of vestibular migraine and benign paroxysmal positional vertigo.

The characteristics of ocular and cervical vestibular evoked myogenic potentials are examined in 26 patients with unilateral Ménière's disease and 15 patients with Ménière's disease and benign paroxysmal positional vertigo (37). In case of frequency amplitude ratio greater than 0,84 , excessive endolymph accumulation is the pathophysiologic factor while if this ratio is greater than 1,79 , the otolithic membrane of the elliptic cyst is damaged by labyrinthus hydrops. The frequency amplitude ratio of Ménière's disease combined with benign paroxysmal positional vertigo can be used as indicator for utricle function evaluation.

The diagnostic criteria for 'vestibular migraine of childhood', 'probable vestibular migraine of childhood' and 'recurrent vertigo of childhood' launched by the Committee for the Classification of Vestibular Disorders of the Bárány Society and the Migraine Classification subgroup of the International Headache Society are described (34).

Twenty-one consecutive vestibular migraine patients are assessed by video head impulse test, electrocochleography and audiometric testing (19). There is a statistically significantly higher proportion of abnormal electrocochleography in Ménière disease than in vestibular migraine $(p=0,02)$ but no significant difference between these two disorders in terms of video head impulse test gain. Only three vestibular migraine patients $(14,28 \%$ of the cases) exhibit summating potential/and action potential values suggestive of endolymphatic hydrops.

The bedside head impulse test is used to differentiate vestibular neuritis from posterior circulation stroke in 38 acute vestibular syndrome patients (17). The accuracy of the bedside head impulse test is only $58 \%$, its sensitivity for vestibular neuritis is high $(88 \%)$, but its specificity is low $(64 \%)$ due to many false-abnormal bedside head impulse tests in posterior circulation stroke $(36 \%)$. The videooculography-supported head impulse test yields excellent specificity (100\%) and moderate sensitivity $(67 \%)$.

In conclusion, additional efforts of the international interdisciplinary scientific community are needed to successfully solve the complex issues of the timely and precise diagnosis of the vestibular disorders as an essential precondition for the sucessful treatment of these patients.

\section{References}

1. Adamec I, Juren Meaški S, Krbot Skorić M, Jažić K, Crnošija L, Milivojević I, et al. Persistent postural-perceptual dizziness: Clinical and neurophysiological study. J Clin Neurosci. 2020;72:26-30.

2. Baloh RW. Vestibular migraine I: Mechanisms, diagnosis, and clinical features. Semin Neurol. 2020;40(1):76-82.

3. Brodsky JR, Lipson S, Bhattacharyya N. Prevalence of pediatric dizziness and imbalance in the United States. Otolaryngol Head Neck Surg. 2020;162(2):241-247.

4. Dlugaiczyk J, Habs M, Dieterich M. Vestibular evoked myogenic potentials in vestibular migraine and Menière's disease: cVEMPs make the difference. J Neurol. 2020;267(Suppl 1):169-180.

5. Domínguez-Durán E, Moreno-de-Jesús C, Prieto-Sánchez-de-Puerta L, Mármol-Szombathy I, Sánchez-Gómez S. Identifying training, diagnostic and therapeutic needs from a comparison in the distribution of vestibular disorders in primary care and in a neurotology unit. Front Neurol. 2020 Nov 20;11:605613. doi: 10.3389/fneur.2020.605613.

6. Dorbeau C, Bourget K, Renard L, Calais C, Bakhos D. Vestibular evoked myogenic potentials. Eur Ann Otorhinolaryngol Head Neck Dis. 2021 Jan 19:S1879-7296(21)00014-4. doi: 10.1016/j.anorl.2021.01.001.

7. Dougherty JM, Carney M, Emmady PD. Vestibular dysfunction. In: StatPearls (Internet). Treasure Island, FL, StatPearls Publishing, 2021.

8. Duarte JA, Leão EM, Fragano DS, Marquez GJ, Pires APBÁ, Silva MLS, et al. Vestibular syndromes in childhood and adolescence. Int Arch Otorhinolaryngol. 2020;24(4):e477-e481.

9. Feil K, Feuerecker R, Goldschagg N, Strobl R, Brandt T, von Müller A, et al. Predictive capability of an iPad-based medical device (medx) for the diagnosis of vertigo and dizziness. Front Neurol. 2018 Feb 27;9:29. doi: 10.3389/fneur.2018.00029.

10. Garone G, Suppiej A, Vanacore N, La Penna F, Parisi P, Calistri L, et al. Characteristics of acute nystagmus in the pediatric emergency department. Pediatrics. 2020 Aug; 146(2):e20200484. doi: 10.1542/peds.2020-0484.

11. Gedik-Soyuyuce O, Gence-Gumus Z, Ozdilek A, Ada M, Korkut N. Vestibular disorders in children: A retrospective analysis of vestibular function test findings. Int J Pediatr Otorhinolaryngol. 2021 Apr 29;146:110751. doi: 10.1016/j.ijporl.2021.110751.

12. Goeldlin M, Gaschen J, Kammer C, Comolli L, Bernasconi CA, Spiegel R, et al. Frequency, aetiology, and impact of vestibular symptoms in the emergency department: a neglected red flag. J Neurol. 2019;266(12):3076-3086.

13. Hülse R, Biesdorf A, Hörmann K, Stuck B, Erhart M, Hülse M, et al. Peripheral vestibular disorders: an epidemiologic survey in 70 million individuals. Otol Neurotol. 2019;40(1):88-95.

14. Hülse R, Warken C, Biesdorf A, Erhart M, Rotter N, Hörmann K, et al. Prävalenz peripher-vestibulärer Erkrankungen bei Kindern in Deutschland. HNO. 2020;68(5):360-366.

15. Ishiwara-Niembro K, Pérez-Rodríguez MA, Navarro-Cervantes FA, Jiménez-Pérez JA. Vestibular and balance disorders in Mexican children and adolescents: Review of 8-year clinical records. Acta Otorrinolaringol Esp (Engl Ed). 2021;72(3):137-142.

16. Li M, Xu X, Qi W, Liang Y, Huang Y, Huang H. Vestibular migraine: the chameleon in vestibular disease. Neurol Sci. 2021;42(5):1719-1731. 
17. Machner B, Erber K, Choi JH, Trillenberg P, Sprenger A, Helmchen C. Usability of the head impulse test in routine clinical practice in the emergency department to differentiate vestibular neuritis from stroke. Eur J Neurol. 2021;28(5):1737-1744.

18. Mahrous MM. Vestibular migraine and benign paroxysmal positional vertigo, close presentation dilemma. Acta Otolaryngol. 2020;140(9):741744.

19. Martines F, Dispenza F, Montalbano C, Priola R, Torrente A, La Gumina R, et al. Comparison of electrocochleography and video head impulse test findings in vestibular migraine and Ménière disease: a preliminary study. J Int Adv Otol. 2020;16(2):183-189.

20. Nowaczewska M. Vestibular migraine - an underdiagnosed cause of vertigo. Diagnosis and treatment. Neurol Neurochir Pol. 2020;54(2):106115 .

21. Özdemir D, Akpınar ÇK, Küçüköner Ö, Mehel DM, Bedir A, Akgül G, et al. Vestibular evoked myogenic potential (VEMP) results in migraine and migrainous vertigo. Acta Otolaryngol. 2020;140(2):140-143.

22. Pan Q, Zhang Y, Long T, He W, Zhang S, Fan Y, et al. Diagnosis of vertigo and dizziness syndromes in a neurological outpatient clinic. Eur Neurol. 2018;79(5-6):287-294.

23. Perez-Carpena P, Lopez-Escamez JA. Do we need to reconsider the classification of vestibular migraine? Expert Rev Neurother. 2021;21(5):503-516.

24. Phillips JS, Newman J, Cox S. Towards providing an automated approach to differentiating the nystagmus of Menière's disease, vestibular migraine, and benign paroxysmal positional vertigo. Otol Neurotol. 2021 Feb 18. doi: 10.1097/MAO.0000000000003083.

25. Rizk HG, Liu YF, Strange CC, Van Ausdal CH, English RC, McRackan TR, et al. Predictive value of vestibular evoked myogenic potentials in the diagnosis of Menière's disease and vestibular migraine. Otol Neurotol. 2020;41(6):828-835.

26. Scarpa A, Gioacchini FM, Cassandro E, Tulli M, Ralli M, Re M, et al. Clinical application of cVEMPs and oVEMPs in patients affected by Ménière's disease, vestibular neuritis and benign paroxysmal positional vertigo: a systematic review. Acta Otorhinolaryngol Ital. 2019;39(5):298-307.

27. Schmäl F. Effektive Diagnostik bei Schwindel und Gleichgewichtsstörungen. HNO. 2020;68(9):703-716.

28. Shimizu K, Imai T, Oya R, Okumura T, Sato T, Osaki Y, et al. Platform posturography of patients with peripheral vestibular dysfunction in the non-acute phase of vertigo. Auris Nasus Larynx. 2021;48(4):577-582.

29. Starkov D, Strupp M, Pleshkov M, Kingma H, van de Berg R. Diagnosing vestibular hypofunction: an update. J Neurol. 2021;268(1):377-385.

30. Strobl R, Grözinger M, Zwergal A, Huppert D, Filippopulos F, Grill E. A set of eight key questions helps to classify common vestibular disorders - results from the DizzyReg Patient Registry. Front Neurol. 2021 Apr 29;12:670944. doi: 10.3389/fneur.2021.670944.

31. Strupp M, Dlugaiczyk J, Ertl-Wagner BB, Rujescu D, Westhofen M, Dieterich M. Vestibular disorders. Dtsch Arztebl Int. 2020;117(17):300310 .

32. Takeuti AA, Fávero ML, Zaia EH, Ganança FF. Auditory brainstem function in women with vestibular migraine: a controlled study. BMC Neurol. 2019 Jun 27;19(1):144. doi: 10.1186/s12883-019-1368-5.

33. Taylor RL, Welgampola MS, Nham B, Rosengren SM. Vestibular-evoked myogenic potential testing in vestibular localization and diagnosis. Semin Neurol. 2020;40(1):18-32.

34. van de Berg R, Widdershoven J, Bisdorff A, Evers S, Wiener-Vacher S, Cushing SL, et al. Vestibular migraine of childhood and recurrent vertigo of childhood: Diagnostic criteria consensus document of the Committee for the Classification of Vestibular Disorders of the Bárány Society and the International Headache Society. J Vestib Res. 2021;31(1):1-9.

35. Vargas-Alvarez A, Ninchritz-Becerra E, Goiburu M, Betances F, Rey-Martinez J, Altuna X. Clinical prevalence of enhanced vestibulo-ocular reflex responses on video head impulse test. Otol Neurotol. 2021 May 14. doi: 10.1097/MAO.0000000000003171.

36. Wang A, Zhou G, Lipson S, Kawai K, Corcoran M, Brodsky JR. Multifactorial characteristics of pediatric dizziness and imbalance. Laryngoscope. 2021;131(4):E1308-E1314.

37. Wang J, Yang L, Zhang J, Han Z, Zhang R, Tang L. Study of ocular vestibular evoked myogenic potentials in benign paroxysmal positional vertigo associated with Menière's disease. Lin Chung Er Bi Yan Hou Tou Jing Wai Ke Za Zhi. 2021;35(2):120-124;130. (in Chinese).

38. Yang TH, Xirasagar S, Cheng YF, Wu CS, Kuo NW, Lin HC. Peripheral vestibular dsorders: nationwide evidence from Taiwan. Laryngoscope. 2021;131(3):639-643

39. Yilmaz MS, Egilmez OK, Kara A, Guven M, Demir D, Genc Elden S. Comparison of the results of caloric and video head impulse tests in patients with Menière's disease and vestibular migraine. Eur Arch Otorhinolaryngol. 2021;278(6):1829-1834.

40. Zwergal A, Dieterich M. Aktuelles zu den häufigen Schwindelsyndromen: Diagnostik und Therapie. Fortschr Neurol Psychiatr. 2021;89(5):211220 .

Reviewer: Assoc. prof. Dr. P. Ruev 